\title{
International carbon market price forecasting using an integration model based on SVR
}

\author{
Linbo Jiang ${ }^{1, a^{*}}$, Peng $\mathrm{Wu}^{1, \mathrm{~b}}$ \\ ${ }^{1}$ School of Business, Sichuan University, China \\ aliborjiang@163.com, bwup.sem@qq.com
}

Keywords: Carbon price forecasting; integration model; SVR; ARIMA; BP neural network; grey system; Genetic Programming

Abstract. Better forecast of carbon emission prices may increase risk control capabilities of emission market stakeholders, and provide decision support for policy makers, financial institutions and enterprises. However, the issue of carbon price forecasting is complicated and several existing prediction models are difficult to achieve satisfactory results. This paper proposes an integration model based on SVR to predict international carbon market price. The model we suggest includes two steps: we respectively establish ARIMA, BP neural network, grey model $\operatorname{GM}(1,1)$ and genetic programming to fit the original sequence of the carbon price at the first phase, and get four prediction results. Additionally, SVR is used to integrate these four results and eventually obtain prediction result. For verification and testing, EUA (DEC15) carbon price from December 3, 2012 to April 10, 2015 under the EU ETS was used to examine the forecasting ability of the proposed integration model. The result demonstrates the accuracy of integration model proposed in this paper is superior to the other three basic models.

\section{Introduction}

The conflict between economic advancement and environmental protection is one of the most serious challenges facing the world today. Since the British government first proposed "low carbon economy" concept in Energy White Paper in 2003[1], various countries have responded, and the transformation from high-carbon economy such as the traditional high energy consumption, high pollution and high emissions to low carbon economy such as the low energy consumption, low pollution, and low emissions has become a consensus. Carbon finance generates as the development of low-carbon economy, and it has now become an important innovation in the financial sector[2].

European Union has taken the active exploration and practice in carbon markets and carbon finance, and first establishes European Union Emission Trading Scheme (EU ETS). During the development process of carbon trading market, the carbon price has always been not only a core issue in the carbon market, but also a hot topic among international and domestic academics.

Nowadays international and domestic academics have done a lot of researches for international carbon price forecasting and proposed many prediction methods. These methods can be divided into single prediction model and hybrid forecasting model according to their characteristics. Single model mainly includes ARIMA, ARCH, GARCH, artificial neural networks, support vector regression, genetic programming methods, etc., which is used to analyze and predict time series of carbon price[3, 4]. However, analyzing and forecasting often may not achieve satisfactory results by using a single model due to economic time series with strong noise and highly sophisticated features. So in recent years, most scholars have used mixed models to research, e.g., Paolella and Taschini[5] and Benz and Trück[6] have used an $\operatorname{AR}(1)-\operatorname{GARCH}(1,1)$ model to fit EUA spot price fluctuations. Bangzhu Zhu and Yiming Wei[7] constructed an ARIMA-LSSVM hybrid model to predict carbon price. Jinliang Zhang and Zhongfu Tan[8] applied a hybrid prediction model based on WT, CLSSVM and EGARCH to the Spanish electricity futures market. G. Peter Zhang[9] presented an ARIMA-ANNs hybrid model while traditional ARIMA model has no effect on digging nonlinear characteristic hidden in a time series. 
In this paper, an integration Model based on SVR to predict International carbon market price is constructed which integrates the respective results of ARIMA, BP neural network, grey system $\operatorname{GM}(1,1)$ model and genetic programming by SVR. We first respectively establish ARIMA, BP neural network, grey model $\operatorname{GM}(1,1)$ and genetic programming to fit the original sequence of the carbon price, and get four prediction results. Additionally, SVR is used to integrate according to the former four results and eventually obtain prediction result.

\section{Methodology}

\section{ARIMA}

ARIMA model is the most common time series model[10], containing AR and MA two parts. The basic idea of ARIMA model is that prediction object time series is viewed as a random sequence, described by specific mathematical model[11]. An ARIMA(p, d, q) model of degree of AR(p), difference(d) and $\mathrm{MA}(\mathrm{q})$ can be mathematically expressed as

$$
\phi(B)(1-B)^{\mathbb{a}} y_{t}=c+\theta(B) \varepsilon_{t}
$$

BP

BP neural network, a multilayer feed-forward neural network trained by backward propagation of errors, is one of the most widely used method of training artificial neural networks[12]. A BP neural network consists of an input layer, at least one hidden layer and an output layer[13]. The training of BP neural network involves a forward process and a backward process[14]. And in the feed-forward process, the signals are calculated through network, and the errors are computed at the output layer. Correspondingly, the weights are updated in the backward process. Technically speaking, BP algorithm calculates the gradient of the errors of the network regarding the network's modifiable weights and finds proper weights that minimize the errors.

Grey model GM $(1,1)$

Grey model can be used to analyze the behavior through little system information when system information is not sufficient[15]. The $\operatorname{GM}(1,1)$ model is a first-order one variable grey model which has been commonly used in prediction. It can simulate system evolution trends by fitting the features of previous observations.

The $\mathrm{GM}(1,1)$ modeling process shows as follows[16]. Assume that the original data sequences are expressed as $X^{(0)}=\left\{x^{(0)}(1), x^{(0)}(2), \ldots, x^{(0)}(n)\right\}$. Let $X^{(1)}=\left\{x^{(1)}(1), x^{(1)}(2), \ldots, x^{(1)}(n)\right\}$, where $X^{(1)}$ is the first-order accumulated generating operation of $X^{(0)}$ [17]. If $X^{(0)}$ and $X^{(1)}$ meet the certain test, then $X^{[1]}$ can be generally expressed as

$$
\frac{\mathrm{d} X^{(1)}}{\mathrm{d} t}+\alpha X^{(1)}=\mu
$$

where the parameter $\alpha$ is the developing coefficient which reflects the developing tendencies of $X^{(0)}$ and $X^{[1]}$ and $\mu$ reflects the variation relationship among data.

Genetic Programming

Genetic Programming (GP) is a common evolutionary computation technique that belongs to the computational intelligence research[18]. Essentially, GP is a set of instructions and a fitness function to measure how well a computer has performed a task.

Specifically, an individual is represented with a GP tree which is built of functions (operators, program statements etc.) and terminals (features, constants etc.)[19]. In order to find possible solutions to a problem, a group of populations are generated by GP in each generation. The first population is usually generated randomly. Subsequent generations are evolved through using genetic operators. Generation by generation, successful populations are evolved in new generations to create improved possible solutions. The quality of solutions is expressed by applying a fitness function[20, 21]. 
Support Vector Regression

Support vector machine (SVM) algorithm is regarded as a supervised learning method that analyzes data[22]. SVM algorithm is used to accurately predict when the data are typically nonlinear, non-stationary. SVM overcomes the over-fitting problem and achieves excellent generalization performance with minimizing the structural risk[23].

A version of SVM for regression is called support vector regression (SVR). The basic idea of SVR is that, the input variables are mapped to high-dimensional feature space $F$ through non-linear transformation and process the linear regression on the $F[24]$. The linear function in SVR is expressed as

$$
f(x)=\omega \cdot \varphi(x)+b
$$

where $w$ denotes the vector of weights and $b$ is the offset from the origin.

According to the structural risk minimization principle, it can be converted to the following optimization problem:

$$
\begin{gathered}
\min J=\frac{1}{2}\|\omega\|^{2}+c \sum_{i=1}^{n}\left(\zeta_{\mathrm{i}}+\zeta_{i}^{*}\right) \\
\text { s.t. }\left\{\begin{array}{c}
y_{i}-\omega \cdot \varphi(x)-b \leq \varepsilon+\zeta_{i} \\
\omega \cdot \varphi(x)+b-y_{i} \leq \varepsilon+\zeta_{i}^{i} \\
\zeta_{i}, \zeta_{i}^{*} \geq 0, i=1,2, \ldots, n
\end{array}\right.
\end{gathered}
$$

In Eq. (4), the parameter $\|\omega\| l$ represents the complexity of the model and $C$ is defined as a regularization variable which penalizes the complex model to avoid over-fitting the training data. The parameter $\varepsilon$ denotes the insensitive loss function and $\bar{\zeta}_{\mathrm{i}}$ and $\bar{\zeta}_{i}$ are slack variables.

\section{Data and Modeling}

\section{Data Source}

In this study, European Union allowance (EUA) (DEC15) futures prices of daily trading from December 3, 2012 to April 10, 2015 from the London Intercontinental Exchange (ICE) were selected to examine the forecasting ability of the proposed integration model. The main reason is that EUA is a kind of carbon finance whose trading volume is the biggest and liquidity is the highest. As is well known, the "Kyoto Protocol" came into effect in 2005, and the EU carbon emissions trading market was formally established at the same year, specifically the first phase is 2005-2007, the second phase 2008-2012 and the third phase 2013-2020[25]. Since various carbon futures contract prices have huge trends at different periods, the data from December 3, 2012 to February 27, 2015 with a total of 572 observations are used as the training set and the data from March 2, 2015 to April 10, 2015 with a total of 28 observations are defined as the testing set. The carbon price trend is shown in Fig. 1.

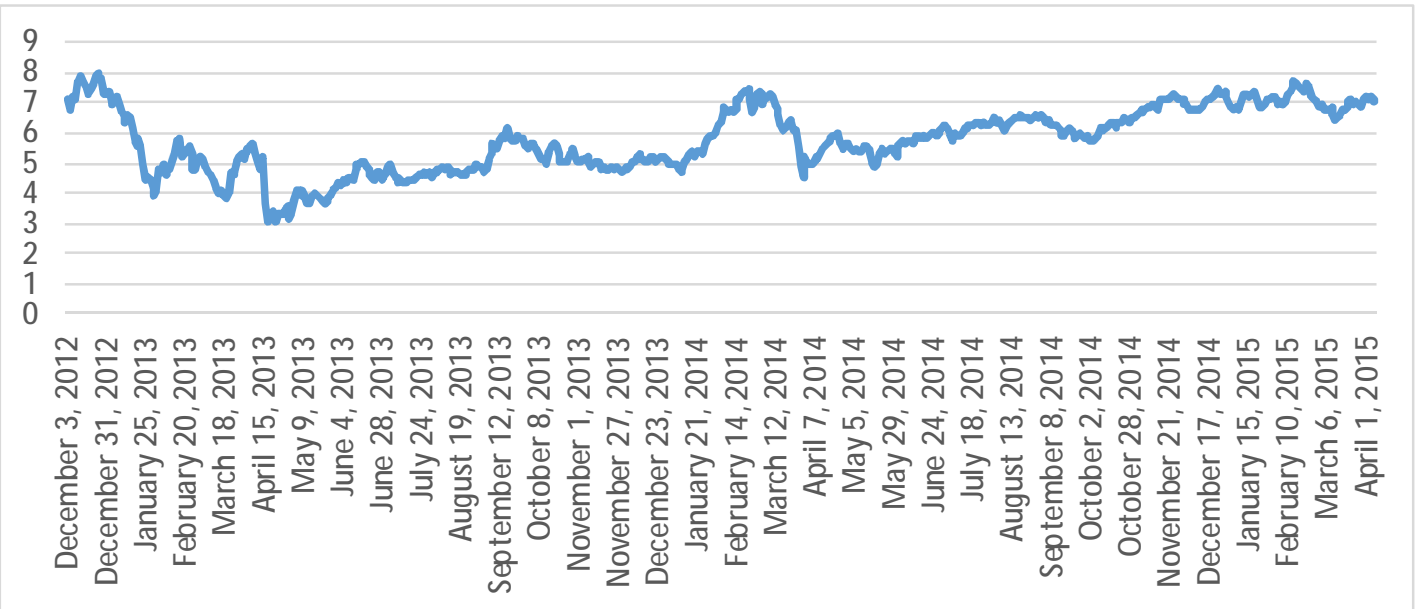

Fig. 1. EUA (DEC15) carbon price for December 3, 2012-April 10, 2015 
Modeling process

In this paper, an integration model based on SVR to predict international carbon market price is constructed, which integrates the respective results of ARIMA, BP neural network, grey system GM $(1,1)$ model and genetic programming by SVR. We first respectively establish ARIMA, BP neural network, grey model $\operatorname{GM}(1,1)$ and genetic programming to fit the original sequence of the carbon price, and get four prediction results. Additionally, SVR is used to integrate the previous four results and eventually obtain prediction result. Fig. 2 describes the modeling process and the specific steps include:

a) The initial processing for original carbon price time series. All data are divided into a training set and a test set, i.e., $y_{t 1}$ and $y_{t z}$.

b) Constructing four basic models. For the training set, ARIMA, BP neural network, grey model $\mathrm{GM}(1,1)$ and genetic programming are built to simulate and predict.

c) Constructing the proposed integration model. Let the former four prediction results at the first phase be $x_{1}, x_{2}, x_{3}$ and $x_{4}$, and they are viewed as independent variables in SVR at the second phase. Thus, carry out integrated forecasting and obtain a final result $\hat{y}_{t}$.

d) The certain tests for the proposed integration model.

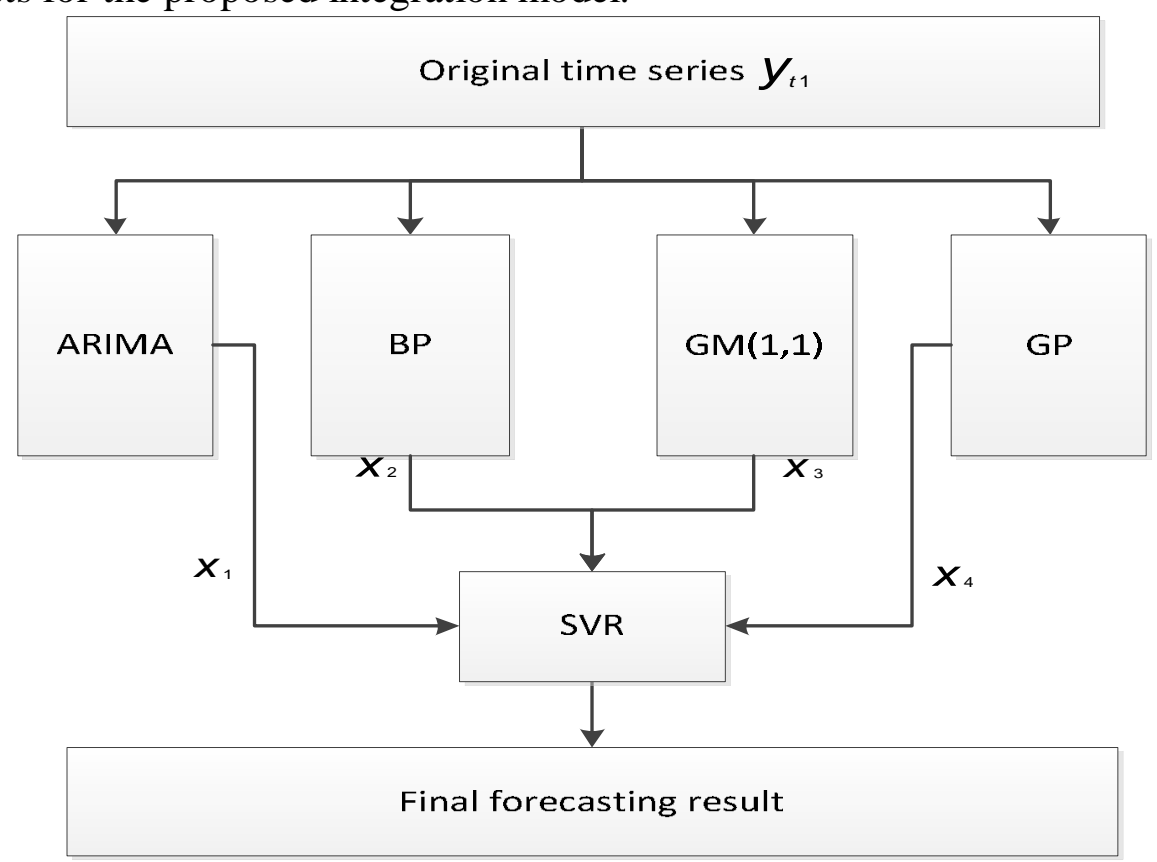

Fig. 2. Modeling flowchart

\section{Results and Conclusions}

All the models are implemented in the R 3.2.0 and Matlab 2011b platform. To measure the forecasting performance, two main criteria are used. The root mean square error (RMSE) and mean absolute percent error (MAPE) are adopted, which are respectively expressed as

$$
\text { RMSE }=\sqrt{\frac{\sum_{i=1}^{m}\left(\hat{y}_{i}-y_{i}\right)^{2}}{m}}
$$

and

$$
\text { MAPE }=\frac{1}{m} \sum_{i=1}^{m} \frac{\left|\hat{y}_{i}-y_{i}\right|}{y_{i}}
$$

where $y_{i}$ is the actual value, $\hat{y}_{i}$ is the predicted value and $m$ denotes the number of sample points. Generally speaking, the smaller the RMSE and MAPE are, the better the forecasting performance is.

Table 1 shows the results from the model comparison for the prediction accuracy. We can see that the proposed integration model outperforms other four basic models due to the smallest RMSE and 
MAPE. And Fig. 3 describes the comparison between the actual carbon price and the final prediction for the test set, which also demonstrates the proposed integration model to predict carbon price is feasible.

Table 1Forecasting accuracy comparison of different models.

\begin{tabular}{|c|c|c|c|c|c|}
\hline model & ARIMA & BP & GM(1,1) & GP & Integration model \\
\hline RMSE & 0.2717 & 0.3896 & 0.1328 & 0.3688 & 0.1074 \\
\hline MAPE & 0.0342 & 0.0450 & 0.0290 & 0.0431 & 0.0186 \\
\hline
\end{tabular}

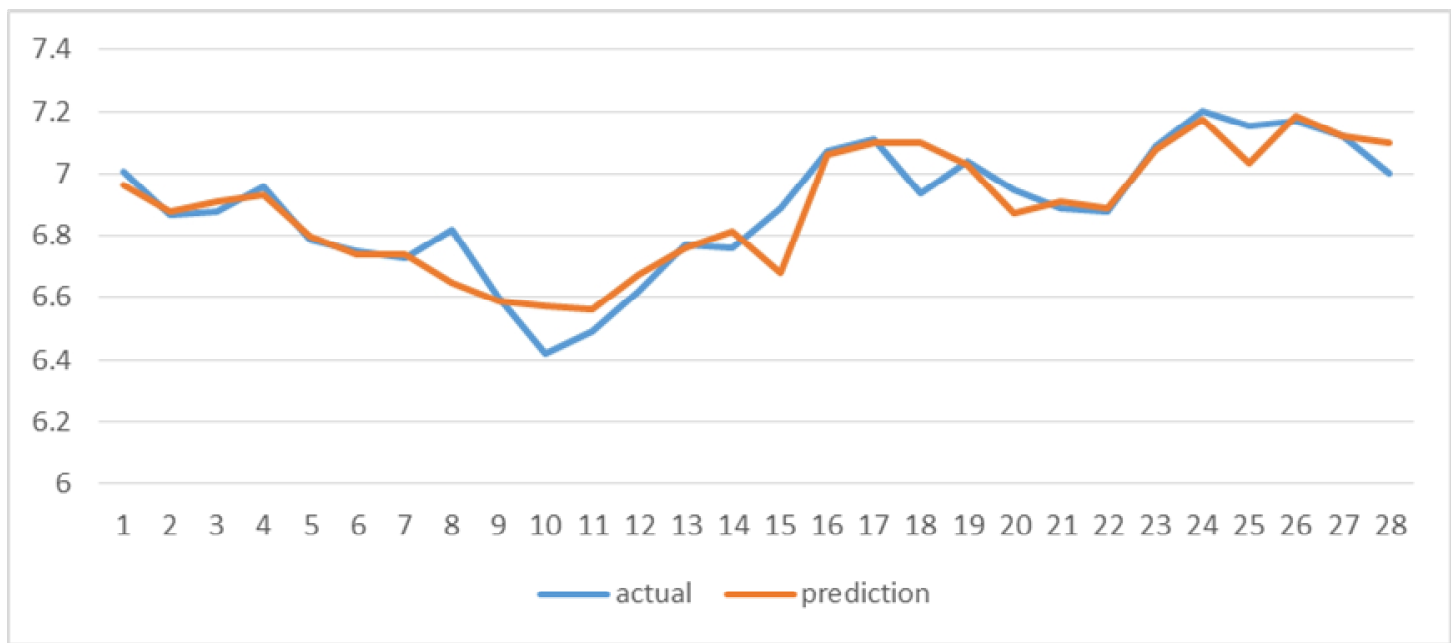

Fig. 3. The final prediction result

The carbon trading markets have been successively established in Shenzhen, Beijing, Shanghai, Tianjin, Guangdong, Hubei and Chongqing since June 2013, which marks the opening salvo in China's carbon emission trade. So accelerating the development of carbon trading market conforming to national condition and improving international carbon price forecasting ability have great realistic significances. Therefore, the proposed integration model in this paper which can achieve superior forecasting performance for carbon price is a promising methodology and provide decision-making references for policy makers, financial institutions and enterprises.

\section{References}

[1] DTI, U., Energy White Paper: Our energy future-creating a low carbon economy. DTI, London (In Chinese), 2003.

[2] Labatt, S. and R.R. White, Carbon finance: the financial implications of climate change. Vol. 362. 2011: John Wiley \& Sons.

[3] Byun, S.J. and H. Cho, Forecasting carbon futures volatility using GARCH models with energy volatilities. Energy economics, 2013. 40: p. 207-221.

[4] Wang, Y. and C. Wu, Forecasting energy market volatility using GARCH models: Can multivariate models beat univariate models? Energy Economics, 2012. 34(6): p. 2167-2181.

[5] Paolella, M. and L. Taschini, An econometric analysis of emission trading allowances. Swiss Finance Institute, 2006: p. 06-26.

[6] Benz, E. and S. Trück, Modeling the price dynamics of CO 2 emission allowances. Energy Economics, 2009. 31(1): p. 4-15.

[7] Zhu, B. and Y. Wei, Carbon price forecasting with a novel hybrid ARIMA and least squares support vector machines methodology. Omega, 2013. 41(3): p. 517-524.

[8] Zhang, J. and Z. Tan, Day-ahead electricity price forecasting using WT, CLSSVM and EGARCH model. International Journal of Electrical Power \& Energy Systems, 2013. 45(1): p. 362-368.

[9] Zhang, G.P., Time series forecasting using a hybrid ARIMA and neural network model. Neurocomputing, 2003. 50: p. 159-175. 
[10] Box, G.E. and G.M. Jenkins, Time series analysis: forecasting and control, revised ed. 1976: Holden-Day.

[11] Lütkepohl, H. and M. Krätzig, Applied time series econometrics. 2004: Cambridge University Press.

[12] McClelland, J.L., D.E. Rumelhart, and P.R. Group, Parallel distributed processing. Explorations in the microstructure of cognition, 1986. 2.

[13] Hamzaçebi, C., D. Akay, and F. Kutay, Comparison of direct and iterative artificial neural network forecast approaches in multi-periodic time series forecasting. Expert Systems with Applications, 2009. 36(2): p. 3839-3844.

[14] Zheng, B., et al., Predictive modeling of hospital readmissions using metaheuristics and data mining. Expert Systems with Applications, 2015.

[15] Deng, J., The primary methods of grey system theory. Huazhong University of Science and Technology Press, Wuhan, 2005.

[16] Li, G.-D., D. Yamaguchi, and M. Nagai, A GM $(1,1)-$ Markov chain combined model with an application to predict the number of Chinese international airlines. Technological Forecasting and Social Change, 2007. 74(8): p. 1465-1481.

[17] Pai, T.-Y., et al., Predicting air pollutant emissions from a medical incinerator using grey model and neural network. Applied Mathematical Modelling, 2015. 39(5): p. 1513-1525.

[18] Koza, J.R., Genetic programming: A paradigm for genetically breeding populations of computer programs to solve problems. 1990: Stanford University, Department of Computer Science.

[19] Langdon, W.B., Genetic programming-computers using "Natural Selection" to generate programs, in Genetic Programming and Data Structures. 1998, Springer. p. 9-42.

[20] Castelli, M., et al., Prediction of Energy Performance of Residential Buildings: aGenetic Programming Approach. Energy and Buildings, 2015.

[21] Tahta, U.E., S. Sen, and A.B. Can, GenTrust: A genetic trust management model for peer-to-peer systems. Applied Soft Computing, 2015. 34: p. 693-704.

[22] Cortes, C. and V. Vapnik, Support-vector networks. Machine learning, 1995. 20(3): p. 273-297.

[23] Kavaklioglu, K., Modeling and prediction of Turkey's electricity consumption using Support Vector Regression. Applied Energy, 2011. 88(1): p. 368-375.

[24] Herman, B.R., B. Forget, and K. Smith, Progress toward Monte Carlo-thermal hydraulic coupling using low-order nonlinear diffusion acceleration methods. Annals of Nuclear Energy, 2014.

[25] Kossoy, A. and P. Guigon, State and trends of the carbon market 2012. 2012. 